\title{
Do Clinical Guidelines for Pressure Ulcers Serve as a Barometer for the Contribution of Nursing Sciences to Evidence Based Pressure Ulcer Management?
}

\author{
Dimitri Beeckman* \\ University Centre for Nursing and Midwifery, Ghent University, BELGIUM
}

Received: September 28, 2015; Accepted: October 03, 2015; Published: November 30, 2015

*Corresponding author: Dimitri Beeckman, University Centre for Nursing and Midwifery, Ghent University, BELGIUM, Tel: +32-9-332-83-48;

E-mail: Dimitri.Beeckman@UGent.be

It is an honor to write this editorial for the inaugural issue of SOJ Nursing \& Health Care. Discussing the eligibility of clinical guidelines to serve as a barometer for the contribution of nursing science to evidence based practice immediately appeared when being invited for this contribution. This topic results from my experience with developing guidelines for the Belgian Healthcare Knowledge Centre[1] and the European Pressure Ulcer Advisory Panel (National Pressure Ulcer Advisory Panel, European Pressure Ulcer Advisory Panel and Pan Pacific Pressure Injury Alliance, 2014) [2]; both on the topic of pressure ulcer management. Choosing pressure ulcers as a case for this editorial is legitimate because they cause an important burden in hospitals, nursing homes and the community. They are associated with considerable discomfort [3], pain [4], and increased mortality [5] and high cost [6]. Pressure ulcers are internationally recognized as adverse outcomes of the admission to a healthcare facility and are key clinical indicators of the quality of care [7]. They are widely perceived as a nursing sensitive outcome, which means that nursing actions are linked to the presence or absence of pressure ulcers [8]. This implies that preventive actions are one of the most frequently applied interventions carried out by nurses. Some of these interventions have a high impact on nursing resources (e.g. patient repositioning) and budgets (e.g. high tech redistributing mattresses).

\section{Evidence- Based Practice and Clinical Guidelines}

Since the early 1990s, Evidence-Based Practice or EBP became hype in nursing. Sacket, one of the pioneers, defined EBP as the conscientious, explicit and judicious use of current best evidence in making decisions about the care of the individual patient. This means integrating individual clinical expertise with the best available external clinical evidence from systematic research [9]. Clinical guidelines are considered to be an important tools to base our clinical practice on current best evidence [10]. They are developed to provide a basis for rational decisions for clinicians and patients about the management of defined pathologies or medical conditions. To develop guidelines we need thoroughly performed research producing valid and reliable outcomes. When developing clinical guidelines, many efforts go to systematically retrieve and critically appraise relevant studies, to assign a level of evidence and to classify recommendations as either strong or weak. Assessing the quality of nursing studies being used in clinical guidelines for pressure ulcer management may give an indication of how nursing science have contributed to evidence based practice in this area.

\section{What did we learn from the pressure ulcer management guidelines?}

During the development of the pressure ulcer prevention guidelines, we learned that mainly the input of expertise from clinical experts led to the development of recommendations; this despite the large number of studies in this domain. The insufficient methodological quality of the studies did not allow the development of recommendations with high level of evidences. As recommended by Sackett in his [9] definition on EBP, we were forced to integrate consensus from clinical experts with the best available external clinical evidence from our systematic search. On this point, some critical reflection and caution are needed. A consensus-driven process with experts, which can be performed systematically and rigorously to some degree, still reflects the uniform agreement among a panel of experts with often predetermined ideas and opinions. Guideline developers should recognize this challenge and do all possible efforts to set- up a formal consensus-driven process leading to valid, reliable and applicable recommendations.

Secondly, nurse scientists seem to keep on making the same methodological mistakes when it comes to pressure ulcer research and they fail to mobilize external funding for large scale studies. It is very likely that both conclusions are interrelated as better research will lead to more funding opportunities. Other possible explanations are the tough economic times for external fundraising, the lack of (inter-) national collaboration between researchers (both for laboratory based research and clinical research), and lack of knowledge about possible funding agencies. Besides, pressure ulcer research (and thus funding budgets) is very fragmented. Joining forces and building on leading international research groups and programmes for 
research will lead to greater opportunities for funding. To date, large scale RCTs are still mainly reserved for topics that get support from pharmaceutical industry, leading to possible high levels of conflict of interest.

Thirdly, the lack of pre-market evaluation of medical devices for pressure ulcer prevention in Europe is an important issue in this domain. As Class I devices are not intended to sustain life or be substantially important in preventing human health impairment, and may not present an unreasonable risk of illness or injury, they do not require clinical trials to be commercialized, hampering guideline developers to formulate recommendations based on evidence about their effectiveness.

\section{What do we need?}

Interventions for pressure ulcer prevention are good examples of complex nursing interventions. As for many other complex nursing interventions, the evaluation of preventive interventions for pressure ulcers should be done systematically in a cyclic process of development, feasibility testing/piloting, and implementation. The role of RCTs should not be underestimated and should really be seen as an essential step in the evaluation process.

As a conclusion, for the case of pressure ulcer prevention,clinical guidelines can serve as a barometer for the contribution of nursing science to evidence based practice to some extent. However, we should be carefull with this conclusion as the area of investigation is very complex (requiring complex and often expensive study designs), funding opportunities are scarce (but should be explored more thoroughly through collaborative initiatives) and industry is having a substantial impact on research activities and outcomes. By writing this editorial I would like to highlight the need of thoroughly performed research in nursing and healthcare in general. I also would like to invite readers to submit their research (of course broader than pressure ulcer management) to SOJ Nursing \&
Health Care and to share knowledge, experiences and in- depth insights with a truly international readership.

\section{References}

1. Beeckman D, Matheï C, Van Lancker A, Van Houdt S, Vanwalleghem $\mathrm{G}$, Gryson L, et al. A national guideline for the prevention of pressure ulcers. KCE Reports. Brussels, Belgium: Belgian Health Care Knowledge Centre (KCE). 2013.

2. National Pressure Ulcer Advisory Panel, European Pressure Ulcer Advisory Panel and Pan Pacific Pressure Injury Alliance. Prevention and Treatment of Pressure Ulcers: Quick Reference Guide. Emily Haesler (Ed.). Cambridge Media: Perth, Australia;2014.

3. Gorecki C, Closs SJ, Nixon J, Briggs M. Patient-reported pressure ulcer pain: a mixed-methods systematic review. J Pain Symptom Manage. 2011;42(3):443-59. doi: 10.1016/j.jpainsymman.2010.11.016.

4. Hopkins A, Dealey C, Bale S, Defloor T, Worboys F. Patient stories of living with a pressure ulcer. J Adv Nurs. 2006;56(4):345-53.

5. Redelings MD, Lee NE, Sorvillo F. Pressure ulcers: more lethal than we thought? Adv Skin Wound Care. 2005;18(7):367-72.

6. Demarré L, Van Lancker A, Van Hecke A, Verhaeghe S, Grypdonck $\mathrm{M}$, Lemey J, et al. The cost of prevention and treatment of pressure ulcers: A systematic review. Int J Nurs Stud. 2015;52(11):1754-74. doi: 10.1016/j.ijnurstu.2015.06.006.

7. Van den Heede K, Clarke SP, Sermeus W, Vleugels A, Aiken LH. International experts' perspectives on the state of the nurse staffing and patient outcomes literature. J Nurs Scholarsh. 2007;39(4):290-7.

8. Jull A, Griffiths P. Is pressure sore prevention a sensitive indicator of the quality of nursing care? A cautionary note. Int J Nurs Stud. 2010;47(5):531-3. doi: 10.1016/j.ijnurstu.2009.12.019.

9. Sackett DL, Rosenberg WM, Gray JA, Haynes RB, Richardson WS. Evidence based medicine: what it is and what it isn't. BMJ. 1996;312(7023):71-2.

10. Woolf SH, Grol R, Hutchinson A, Eccles M, Grimshaw J. Clinical guidelines: potential benefits, limitations, and harms of clinical guidelines. BMJ. 1999;318(7182):527-30. 